\title{
Topics in Structural Chemistry through After-Dinner Humor
}

\author{
Angelo Gavezzotti*[a]
}

Abstract: Some of the best stories told by Jack Dunitz in after-dinner speeches introduce comments and personal views on aspects of contemporary structural chemistry: crystal structure analysis and prediction, facts and fallacies in the study of intermolecular bonding, and theories of crystal formation, from nucleation to growth. The role of X-ray crystallography in the determination of the structure of matter at a molecular level is highlighted, with broad and pervasive bearings on present-day applications in materials science. A short essay presents the results of molecular simulations on the stability of small molecular clusters with crystalline structure: the future seems to lay not in the analysis of crystal structures after they are formed, but in the study of autocatalytic insurgence of long-range ordering during nucleation processes.

Keywords: crystal growth $\cdot$ crystallography $\cdot$ history of science $\cdot$ Jack Dunitz $\cdot$ simulations

\section{Foreword}

Humor is what makes Homo sapiens different from all other animals. There is no humor without self-consciousness, of which animals are notoriously deprived, perhaps with the exception of some house cats. Humor confers a certain lightheartedness to human life, a lifeboat in this valley of tears, and scientific life is no exception. Jack David Dunitz (henceforth JDD) has always been ready to find the humorous side of everything, including his and other people's science. He entitled one of his papers Making the Best of a Bad Job $;^{[1]}$ after receiving adverse refereeing on a paper based on Cambridge Database crystal structures, where the key objection was "the work is not based on original data", he answered the editor by saying that in the same spirit, one would have rejected Mendeleev's paper on the first Periodic Table.

It would be almost impossible, and also useless, to provide one more account of JDD's scientific achievements in one short paper. I have therefore chosen to present an account of his storytelling abilities, linking the keen spirit of those stories to moments and topics in contemporary structural chemistry, with special emphasis on JDD's particular field of enterprise, the molecular and crystalline structure of organic compounds. Many of us recall the times when, at meetings and conferences, after-dinner speeches were a regular and expected feature. After hearing the tinkle of a spoon on a glass, a great moment was when one saw JDD stand (Figure 1) ready to deliver what one knew for certain would be a good story. Most of which were based on lateral thought, the ability of seeing ordinary things from an unusual perspective, or were based on logical twists, as most good stories are, especial- ly of the kind of quick, point-blank salvoes that sometimes go under the name of "Jewish humor". ${ }^{2]}$

As the title of the series is "Rosarium Philosophorum", I have taken the liberty of using here and there terms and paragraph headings from ancient logic or philosophy. Although "Rosarium" has an echo of Rosicrucian terminology, I apologize for not dwelling on it, mainly because humor and mysticism are at absolute odds with one another.

\section{Where Are We? JDD's First Story ${ }^{[3]}$}

"Two men in a balloon are lost in thick fog and decide to land to ask for directions. As they hover on a large courtyard among tall buildings, they see a gentleman going by. So they ask: 'Excuse me, sir, where are we?'

The gentleman looks up at them, ponders for a moment and then says: 'Well, you're in a balloon.'

The two balloonists look at each other, then one says: 'I know where we are; we're in Rehovot at the Weizmann Institute!'

'Why? says the other.'

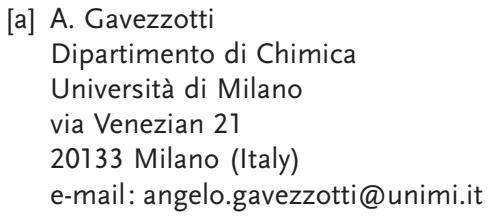

a] A. Gavezzotti

Dipartimento di Chimica

via Venezian 21

e-mail: angelo.gavezzotti@unimi.it 


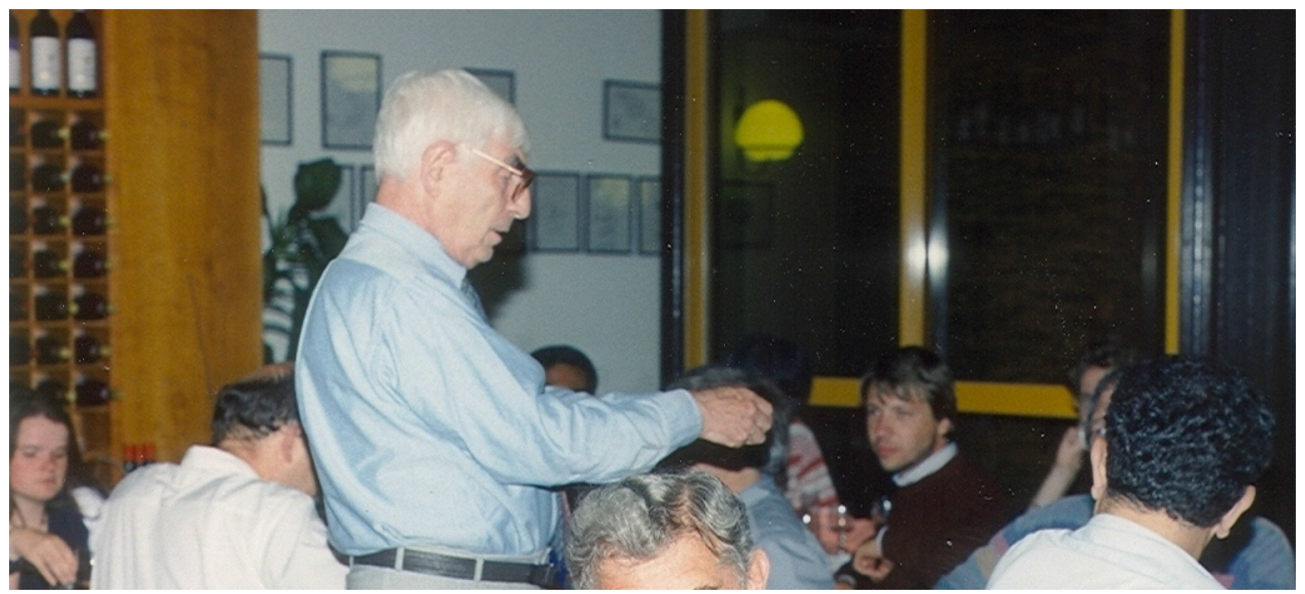

Figure 1. Jack Dunitz working the after-dinner crowd at the NATO ARW in Sestri Levante, $1995 .{ }^{[3]}$ See www.iucr.org/gallery/1995/nato-arw for more pictures.

'Because that gentleman's answer is one hundred percent correct and totally irrelevant."'

(Of course any other respectable institution can take the Weizmann's place in the story, according to time and place of storytelling.)

\subsection{Gloss $^{[4]}$}

There are many examples in teaching and in written science when a truism is mistaken for an explanation. For example, many teachers explain that a liquid crystallizes because the free energy of the solid becomes lower than that of the liquid, and many paper authors take some time to declare that some polymorphic crystal form is more stable because its free energy is lower than that of other phases. Having a lower free energy is, in fact, the definition of being thermodynamically more stable, so such sentences are actually parallel statements, rather than consequential explanations.

\subsection{Scholium: ${ }^{[5]}$ The Mystery of Crystallization}

We do not actually know why a liquid crystallizes. But "why" is a scarcely scientific adverb, seeming to imply the existence of an undisputable truth, and the chances of heading towards some form of circular argument increase. The question should be rephrased into "how" does a liquid crystallize, or to be more specific, the question should be answered by proposing a structural, energetic, and kinetic model of crystallization, susceptible of amelioration and even of complete reformulation in view of new evidence. Such a model is precisely something that at the moment of writing still escapes even the most perceptive structural chemists.

Early models of crystallization were built upon extremely simplified constructions, invoking the assembling of cubic elements - in organic chemistry, a cubic model of a molecule looks pretty much like the (in)famous "spherical horse" model: a physicist stating that she would be able to set up a mathematical equation of a horse galloping, but only assuming a spherical horse. A school of thought called classical nucleation theory (CNT) postulates structureless early nuclei, working with diameters (spheres, again...). Correlating these platonic constructs with surface free energy, the theory often ends up with equations for nucleation velocities that include a large number of distasteful $\pi$ s and scarcely palatable numerical coefficients. ${ }^{[6]}$ A pedantic physical chemist might even insinuate that "surface free energy" is an oxymoron, because thermodynamic functions like enthalpy and entropy are rigorously defined only for infinite and perfectly homogeneous systems. Besides, the perversity of exponential dependence (sometimes called the curse of thermodynamics) makes it so that even minor free energy changes burst into differences of tens of orders of magnitude among equilibrium constants or nucleation frequencies. Classical nucleation theory has been called "the only scientific discipline in which agreement within nine orders of magnitude is considered a success. ${ }^{[[]]}$

\subsection{Argumentatio ${ }^{[8]}$}

Consider a beaker with a concentrated solution of molecule $\mathrm{A}$ in solvent $\mathrm{B}$. At a given point in time, temperature, and concentration space, a bell rings and molecules of A invariably find their way to a solid, compact, and long-range ordered construction called a crystal. The path from dispersed molecules to ordered arrays is one in millions of billions possible ones through phase space, but molecules find it without hesitation, driven by a complex administering of kinetic and thermodynamic instructions encoded in their electron distribution. The reader who wants to see this miracle happen can dissolve a spoonful of succinic anhydride in any solvent of his choice, warm 
up the solution a little in a test tube, and then let cool: wham! bright transparent needles will grow in no time at all, and they are always the same $P 2_{1} 2_{1} 2_{1}$ crystal structure, no matter how hard one tries to get something else.

An old adage says that prediction is a difficult exercise, especially when it concerns the future. Imitating an old, mythic master, I could say, "Et graviter frendens sic fatis ora resolvit": ${ }^{[9]}$ here's my prediction for the future of nucleation science, a topical aspect of crystal structural chemistry. It will become more and more clear that crystal nucleation from solution is a two-step mechanism, ${ }^{[10]}$ by which solute molecules first coalesce into liquid-like droplets, which grow until a critical size is reached. At that point, the exploration of phase space becomes restricted to a certain number of favorable interlockings that win over less favorable molecular adhesion modes. Crystalline long-range order begins to set in by a largely autocatalytic, self-replicating, kinetically dominated mechanism. The followers of this line of thought contend that the critical size must be of the order of thousands of molecules, so that the above process may proceed in a sizeable core region protected from the structural vagaries of surface molecules. Order thus propagates from the center of the nucleus outwards, as the increasingly large "good" particle zooms through the system like a doomsday machine, phagocytizing molecules from the less successful micelles that are unable to grow. When the crystalline nucleus reaches a macroscopic size, that is, a size in which the disordering influence of surface molecules becomes negligible, the proceedings change over from nucleation to actual crystal growth. How many may there be of these nucleation sites? At the beginning, quite a large number; towards the end, very few, if one considers that starting with Avogadro's number of molecules one usually ends up with a batch of a few tens of crystalline individuals.

The persistent formation of only one crystal form is a frequent course of events with organic compounds, but unfortunately, many other occurrences are also common. Most dismayingly, sometimes, one gets no crystals at all; no incipient nuclei are able to perform the devastating journey through the system, required to grow at the expense of others, and the solution stays dispersed with submicroscopic, unproductive micelles. Otherwise, instead of crystallization, one may get oils, or "earwax" (a colorful expression pointed out to this author by Peggy Etter); the separation of solute from solvent has passed the stage that one may call demixing, but for some reason, the final stage that induces long-range order cannot be reached and the molecular aggregate stays in a sort of structural limbo between liquid and amorphous solid. On other occasions, one may get a mixture of different polymorphic crystal forms, because more than one precursor is able to survive in sizable amounts. This model may be checked against the multiform events that occur when one tries to get well-shaped single crystals from solution; each syn-

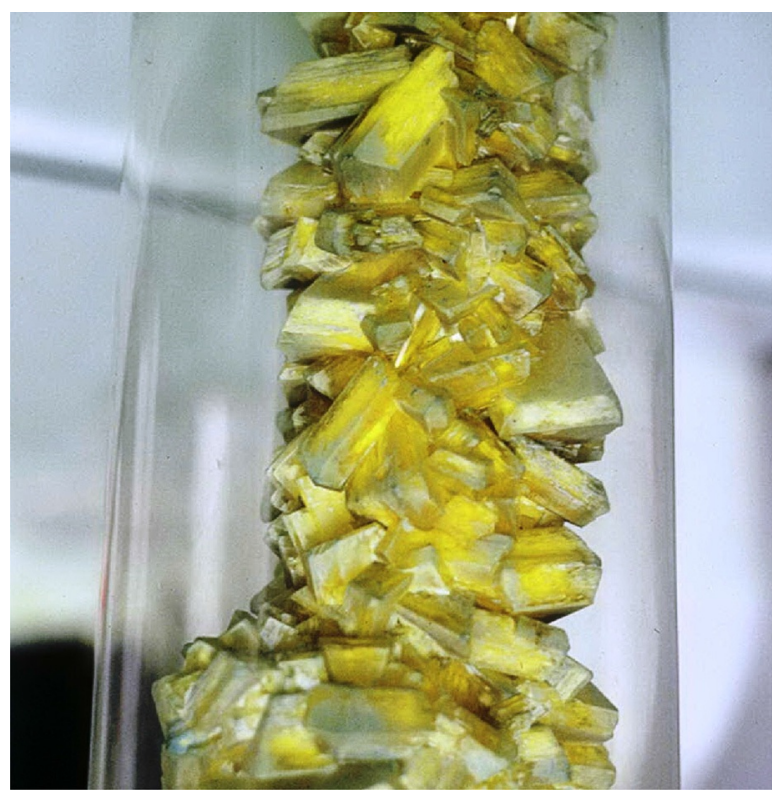

Figure 2. Monster crystal agglomerates (probably a sulfate of a polysubstituted nitrobenzene or nitrotoluene) crystallized in the lab of Ludwig Koerner at the School of Agriculture in Milano. The largest individuals are some $7 \mathrm{~cm}$ across. Koerner, famous for the chemical proof of the equivalence of bonds in the benzene ring, was a notorious crystallization maniac. ${ }^{[1]}$ Picture taken by the author by courtesy of departmental staff.

thetic organic chemist has his store of such (horror) stories. So, prediction and control of crystal formation is one of the long-standing riddles of crystal structural chemistry. Crystallization is often an art rather than a science (Figure 2).

\subsection{Confirmatio or Confutatio ${ }^{[8]}$}

I can imagine a JDD-type, tongue-in-cheek comment on the above prediction in crystal nucleation theory: "this is at least less preposterous than any other theory I've heard on the subject."

The experimental difficulties in studies of crystal nucleation for organic compounds stem from the difficulty of catching a revealing, differential beep from already organized molecules. The case lends itself to a comparison between Gargantuan claims by physicists and the Lilliputian quandaries of the chemists: they can measure (how and if, it's anybody's guess) gravitational waves by interference between two signals, one going through four miles and the other through four miles and a quarter of a millimeter, while we chemists cannot tell, say, the difference between an NMR signal from a molecule loose in solution or trapped in an incipient crystal. Hence, there are chances that confirmation or confutation of the above argumentatio may come sooner from molecular simulation than from experiment. On the computational side, there have been bold attempts to force a liquid system 
through phase space towards the crystalline state, by imposing a structural bias to the usual Metropolis criterion in a Monte Carlo-like method $;^{[12]}$ unfortunately, the procedure works only for the $P 1$ space group, because a mathematical form for the structural bias can be simply written only for the lineup of parallel molecules. Some promising developments are coming by a highly refined, recursive molecular dynamics procedure ${ }^{[13]}$ that drives the system to a fast exploration of phase space. A big advance would be made if somebody were able to design an "order parameter" expressed in a single number that could reflect the amount of long-range periodicity in a high-symmetry space group. Such a number could be fed into a computer and maximized computationally. Note that in organic crystal chemistry $P 2_{1} / c$ is a high-symmetry space group, while in inorganic and mineral crystal chemistry, trigonal and cubic space groups are common.

In my view, the future of the structural chemistry of crystal constitution, or rather, of the fun of it, at least from the molecular modeling perspective, is in the investigation of molecular mechanisms presiding over the early stages of molecular coagulation from liquid to crystalline solid. That would require a computational lens with a magnification power greater by orders of magnitude than the ones we have at hand today, but also a great deal of imagination in devising the computational experiments, and of intuition in deriving conclusions from their results. It is very seldom the case that a molecular simulation does offer definite proof of chemical evolution paths. Molecular modeling is a powerful thought-provoking tool, and its primary function is to provide inspiration for chemists to express fresh views on old problems. Just what it takes.

\section{Where Are We? JDD's Second Story ${ }^{[3]}$}

"The Prince of Wales (it doesn't matter which particular one) is on a survey trip with a reconnaissance party in a remote part of one of the less commendable Commonwealth possessions. The Prince picks up a complicated piece of instrumentation with lots of lenses, mirrors, and graduated scales, performs some complex calculations, and finally says to his aide-de-camp: 'As I reckon, we're now right on the top of that cliff over there."'

(Variant: they are on a ship and it is the captain that says, "Your Highness, according to your calculations, we are just entering the Westminster Abbey.")

\subsection{Thema: Fluorine Amplificatio ${ }^{[14]}$}

When somebody told him that the craters on the Moon had already been seen by others, Galileo replied something like, "Yes, but they did not know what they were seeing." In science, discovery is not all, what matters even more is consciousness of discovery and promptness in providing evidence and ensuring reproducibility. First of all, of course, one should keep his feet firmly on the ground and be self-conscious about where one stands, without sending out misleading signals. Not always an easy task.

Once asked to describe the chemical behavior of fluorine in an intermolecular context, JDD offered the following paragon: "If fluorine could speak of its social attitudes, it would probably say: 'I hate everybody, irrespective of nationality, race, sex, or creed."

It is a matter of fact that your pancakes do not stick to Teflon-coated pans. For a more scientific expression of the same concept, see the paper which was supposed to put the end mark to a long dispute about the hydrogenbonding abilities of $\mathrm{C}-\mathrm{F}$ fluorine. ${ }^{[15]}$ In spite of its high electronegativity, or maybe just because of that, fluorine is a "hard" atom, with its electrons held tightly close to the nucleus and very scarcely inclined to mate with any other electric entity around. In structural formulae on paper, atoms are often drawn with the usual $\delta+$ or $\delta-$ marks near the symbol element; naive chemistry pictures fluorine with a large negative electric residual, and concludes that it must easily come to Coulombic good terms with positively charged hydrogens. Elementary quantum chemistry reveals that the charge polarization along a $\mathrm{C}-$ $\mathrm{F}$ bond is not so high: the residual charge on fluorine is some 0.19 of an electron in a parametric fitting of the electrostatic potential of fluorobenzene from an MP2/6$31 \mathrm{G}^{* *}$ wavefunction. Besides, good atom-atom interaction, Coulombic or dispersive in nature, requires some electron delocalization in between the bonding termini something that fluorine, the electronic miser, the social misfit sipping its Martini at the end corner of the bar and not speaking to anybody, cannot provide. It is another matter of fact that the parameterization of fluorine in empirical or semiempirical schemes for the computer simulation of organic matter has always been a special problem. $\mathrm{C}, \mathrm{N}, \mathrm{O}-\mathrm{a}$ rather smooth sequence; $\mathrm{O}$ to $\mathrm{F}-$ an abyss. In spite of all that, there is a vast literature ${ }^{[16]}$ on the miraculous intermolecular virtues of fluorine, often based on subjective sorting of minor features of crystal structures of large molecules in which fluorine supplies no more than $5-10 \%$ of the electron load. In this latter case, Luke's $(6: 39-42)$ remark applies, as one is making much of a speck while forgetting the logs. ${ }^{[17]}$

Prejudice and uncritical thinking are a cocktail that many scientists, some of them even respectable, seem to often enjoy. The intellectual malfunction called prejudice puts you above the facts and makes you think that your feet are standing on the top of a cliff a mile apart from where you're actually standing: a modern version of ipse dixit, and add to it that "ipse" in this case does not mean Aristotle (who after all had some merits), but the operator himself. There is no prejudice without uncritical thinking, but there is also unaccompanied uncritical thinking, sailing happily through scientific seas, especially when 
these seas have become a muddy ocean in today's publishing policies.

\subsection{Formal Logic: Simple Syllogism, Barocho and Baralipton}

Clear and unbiased reasoning is a cornerstone of the progress of science. The mechanics of reasoning is called logic. Have you ever asked yourself whether seeing a white dove helps confirming that all crows are black? Well, as a scientist you should have (the answer is no). Matters of this kind were the fodder of scholastic logic, a tortuous maze of recipes for constructing and checking the consequentiality or contradictions of true/false, and/ or/either statements. Scholars were forced to swallow and memorize these recipes, two of which are named as the headers of this section, and to apply them blindly. How lucid these prescriptions have been is demonstrated by the fact that an artistic and architectural epoch that was not famous for its linearity was named after one of them. Nowadays, one is no longer forced to think in barocho; journal editors just ask referees to tick the "do-conclusions-follow-from-evidence" box. But a careful consideration of logic in one's reasoning, when planning an experiment or a modeling calculation, and above all, when writing the conclusions section of a paper, is still a Kantian "categorical imperative".

Serious and systematic attempts at probing the crystal nucleation mechanisms at a molecular level would require careful and ingenuous planning, with ultra-sophisticated instrumentation, or unheard of computing power, or both, along with confidence in your project by some funding agency ready to invest an inordinate amount of money. There are, however, some ways of at least pricking the surface of that monumental problem, using modesty and a modicum of logic. Previous, long experience has shown that the formation of sizeable crystal nuclei happens on a timescale that is way out of reach of straightforward molecular simulation; in other words, preparing a molecular model system in the liquid state, feeding it into a computer, along with a simple Monte Carlo framework and an optimized force field, and waiting for crystallization to happen "spontaneously", is hopeless. Without necessarily being as acute as Saint Thomas Aquinas, the recognized master of scholasticism, a much simpler reverse computational experiment may proceed via the following steps:

1) choose a chemical species (acetic acid) small enough for the introduction of a sizeable number of molecules in your model, and prepare a cluster of $N$ molecules in their crystalline structure;

2) run Monte Carlo simulations at room temperature, and check if the molecules lose the ordered structure and evolve to a liquid-like structure using rotational correlation functions;
3) if orientational correlation is quickly lost, a nucleus with less than $N$ molecules cannot be the initiator of crystallization;

4) repeat the above steps for a $N$-atom crystal cluster surrounded by solvent molecules (water), and compare the loss of rotational correlation for the in vacuo and the solvated cluster; and

5) use further logic to derive some conclusion about stability and the role of the solvent.

Figure 3 shows the results for the in vacuo clusters: they decay to a liquid-like state (rotational correlation decaying to zero) in no time at all, even though the time for loss of correlation increases slightly with the size of the cluster, as expected. (Although time is not a variable in Monte Carlo runs, as an order of magnitude, a million MC steps are the equivalent of a couple of picoseconds). Figure 4 is an impressive picture of the effects of "thermalization", that is, allowing temperature to work out its dynamic effects on a molecular system, on a cluster of 169 molecules of acetic acid.

The results for a solvated cluster (step 4 above; 93 acetic acid molecules +1730 water molecules) show that the time for decay to a randomized structure is much longer than in vacuo, demonstrating that the solvent exerts a considerable stabilizing power - or, in classical terms, that the acetic acid-air surface tension is much smaller than the acetic acid-water one. In any case, by a simple syllogism, the overall outcome of the computational experiments proves that a stable nucleating cluster must be larger (probably much larger) than 93 molecules.

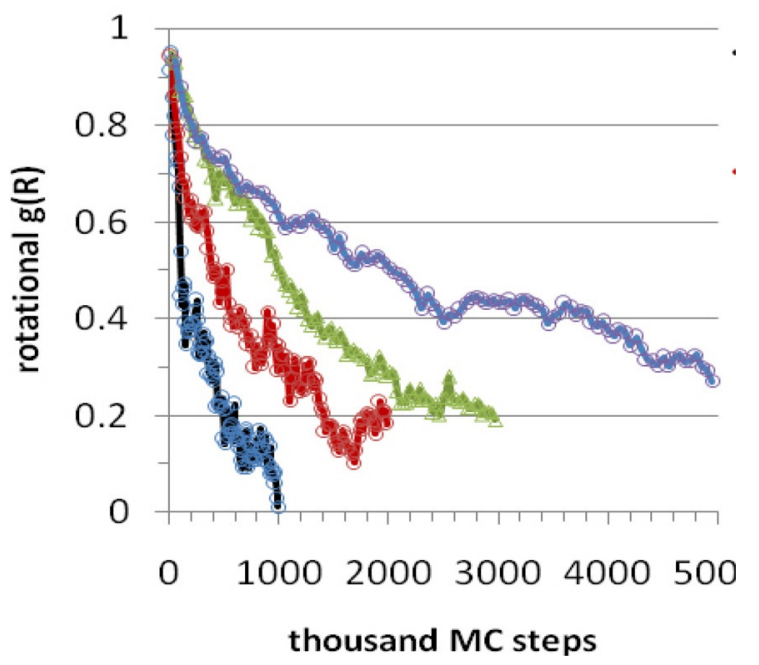

Figure 3. Evolution of rotational correlation functions for clusters of 40,58, 93, and 169 molecules (from left to right) of acetic acid in vacuo. In a perfect infinite crystal, $g(\mathrm{R})$ stays equal to 1 for any simulation length because molecules preserve their relative orientation. $g(\mathrm{R})$ going to zero means total loss of orientational "memory" during the simulation, the computational signal of liquefaction. For the water-solvated 93-molecule cluster, the decay time is much longer. 

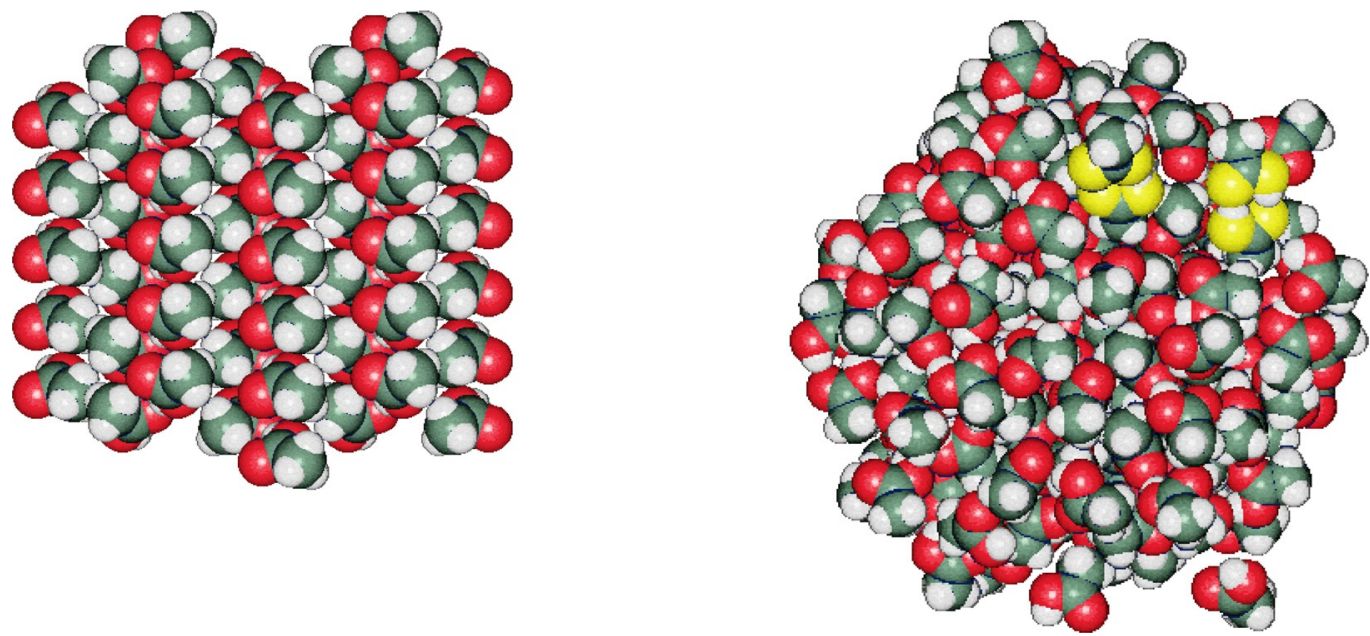

Figure 4. Left: the original 169-molecule acetic acid crystal cluster; hydrogen bonding is in chains (the catemer motif). Right: after 5 million MC steps. Some of the molecules in this completely disordered system have gone back to the favorite double-cyclic motif (pairs highlighted in yellow). Note the considerable increase in size of the cluster. Total cohesive energies are -41.8 and $-39.5 \mathrm{~kJ} \mathrm{~mol}^{-1}$, respectively, with a minimal loss from crystal to liquid state. Graphics by Schakal (E. Keller, University of Freiburg, http://www.krist.uni-freiburg.de).

The simulations could be repeated with other molecules, other solvents, and other cluster sizes, opening up an enormously large field of investigation about the necessary size for a crystal nucleus to be stable. I suspect that the answer would be different in each different case.

\section{Getting Lost and Finding the Way: JDD's Third Story ${ }^{[3]}$}

"A large station wagon pulls into Twin Oaks (pop. 47), Michigan upper peninsula. The driver sees an old man in working clothes and straw hat sitting on a rocking chair in the porch of an old frame house. The driver asks: 'Do you know the way back to Saginaw?'

'Nope.'

'Do you know how to return to Interstate 65?'

'Nope.'

'Do you know at least how to get to a road with traffic signs?'

'Nope.'

Somewhat angrily, the driver blurts out: 'Gee, you don't seem to know much!'

'Nope. But I'm not lost."'

\subsection{Argumentatio: The Way from Molecule to Crystal}

Are we lost in a forest of disperse molecules or can we find our way back to civilization? That is, can we predict a crystal structure from molecular structure? For organic compounds, there have been separate answers in separate times from this author ${ }^{[18]}$ and from JDD. ${ }^{[19]}$ In 2000 , the Cambridge Crystallographic Data Center took the burden of organizing a blindfold test of computational crystal structure prediction. Three crystal structures were determined by X-ray crystallography and were secreted by the organizing committee. A number of computational chemists were given molecular diagrams and were asked to submit their three best predicted crystal structures for each of these molecules. Scheme 1 shows one of them, a small bicyclic compound. This author had written a crystal structure predictor program (PROMET, modestly called "polymorph generator",[20]) and Jack Dunitz, helped by Bernd Schweizer, decided to use that software to try his luck with that molecular midget. The crystal structure was predicted correctly as being the most stable and the one with the smallest deviation from experiment of the whole blind test lot. But there were some backstage secrets. My program came with a detailed manual, which clearly explained that the built-in force field had been developed to run without explicit Coulombic terms. But neither Jack nor Bernd read the manual (or, if they did, they paid no attention), and they added point charges derived by some population analysis on a molecular orbi-<smiles>c1occ2c1CC2</smiles><smiles>C1CNC1</smiles><smiles>Cc1ccc(S(=O)(=O)C2CN=C(c3ccc(NC(=O)OCc4ccccc4)cc3)S2)cc1</smiles>

Scheme 1. Some compounds proposed for the Cambridge crystal structure prediction blindfold tests. From top left, clockwise: the small compound whose crystal structure was predicted by Dunitz and Schweizer; a small molecule whose crystal structure nobody was able to predict; and a very large and flexible molecule correctly "crystallized" by two groups. 
tal calculation. The result was an unheard of, non-optimized force field! Besides, before submission of the predictions, Jack had come to Milano for a meeting, and we discussed his results. He said that they had three or four crystal structures with lattice energies within $0.5 \mathrm{~kJ} \mathrm{~mol}^{-1}$. He asked me what my feeling was about the situation. I said, "Well, play by the rules and submit the most stable structure" - as it turned out, it was "most stable" by just $0.1 \mathrm{~kJ} \mathrm{~mol}^{-1}$.

A community of computational chemists have been playing that game for some fifteen years now, ${ }^{[21]}$ with impressive if contradictory results; witness the comparison between the smallest and the biggest compounds ever proposed (Scheme 1). Progress is coming only at the price of a booming increase in the deployment of computational resources. The thrill of hitting the correct target is simmering in a slurry of hundreds of parallel processors and of petaflops. The percentage of correct predictions churned out by what look like monster computing machines grows at a steady, but still slow, pace. Besides, correct predictions have sometimes been made on laptop PCs with limping force fields, while top quantum chemistry and top-notch computers have scored quite a number of shameful misses. Incidentally, what is the "correct" experimental crystal structure of an organic compound? Here is the transcript of a question-time dialogue after presentation of some results of computational crystal structure prediction: Speaker: "We did not match the known experimental crystal structure for this compound, but our predicted structure is probably a more stable polymorph that has not yet been discovered." From the audience (Christer Aakeroy): "Then how come that when you match the known structure you do not point out that there could be a more stable one that has not yet been discovered?" Saint Thomas would have giggled at this display of scholastic logic.

\subsection{Scholium: Crystal Structure Prediction vs. Crystal Engineering}

Some people, like the man sitting on his porch, can be perfectly at ease by knowing just the essentials. Of course, his example should not be followed by scientists, for whom a broader Weltanschauung is as essential as the local essentials. The possibility of predicting a crystal structure from molecular structure is obviously of great interest for pure science and for applications in all fields where solid-state properties are important. A non-exhaustive list includes materials in general, from plastics to conducting polymers for lightweight electronics; materials for display devices; pharmaceuticals, for bioavailability and solubility, and for polymorph control; agrochemicals for vapor pressures and decay times; pigments for the influence of inner structure on reflecting/absorbing properties. In the chocolate industry, people would very much like to make sense of the mess made by phase transitions of crys- talline fats. If prediction power is limited, even more problematic is control. Still hovering in the crystal structural chemistry sky are the prophetic words in a 1981 paper by Paul and Curtin" [22]: "We seem to be some time away from being able to control or even to predict with real assurance the packing a compound will adopt when it crystallizes, or the relationship of the crystal symmetry to molecular structure." In the 35 years from then, much progress has been made, but the full goal is still far away.

Not to be confused with crystal structure prediction is crystal engineering, a way of saying that should define a multiform (to say the least) discipline whose aim is the design of organic compounds, such that they will adopt a predictable crystal structure. ${ }^{[23]}$ There is a subtle semantic difference, but an wide conceptual divide, between the two approaches. Prediction refers to a chemist "having" a molecule useful for some chemical purpose, and wanting to know how it will crystallize. Engineering refers to a chemist "planning" a molecule and pretending to be confident about how it will crystallize. The two views have one thing in common: in both, success has been sporadic and not entirely reproducible. The unadorned truth is that the intermolecular energy and contact landscape for organic molecules, with their tortuous shapes and multiform electronic structure, is, in general, too complex to be described in terms of a few localized bonds of local interactions. Accordingly, crystal polymorphism is widespread; ${ }^{[24]}$ the same compound can produce as many as eight or so different crystal arrangements, ${ }^{[25]}$ and a single compound, sulfathiazole, over one hundred crystalline solvates. ${ }^{[26]}$ Moreover, many organic compounds, even if endowed with well-recognized synthons, do not crystallize at all. When a predicted crystal structure becomes known experimentally, there are also no strict rules for sorting out which, in fact, are the constituting synthons, against those postulated a priori.

\subsection{Glosses to the Scholium}

\subsubsection{Soft Matter}

Somewhat contrary to the popular belief that crystals are solid and resistant structures, crystalline matter whose constituent is an organic compound makes for flexible, voluble, mutable constructions. With the possible exceptions of salts and zwitterions, organic crystals at room conditions are pretty close to their melting point, and even a modest input of thermal or mechanical energy can produce conspicuous structural consequences. A rather common occurrence is a molecular rearrangement, rotation, or translational displacement, resulting in partial loss of long-range periodicity; in turn, this may entail an increase in the number of independent molecules in the asymmetric unit, or a formal change of lattice symmetry or space group, possibly with a typical (and revealing) doubling of one cell dimension. The new phase is, struc- 
turally speaking, a degradation of the proper crystalline construction, a sort of low-grade specimen on its way to the premelting or melting catastrophes, but automated diffractometer software spells out a new crystal structure and X-ray crystallographers may label it as a new "crystal polymorph". Quite incidentally, the discovery of new polymorphs has recently become a matter of consequence because of the dangers related to patent infringement of pharmaceutically active ingredients. There has been, therefore, a sort of race to claim new polymorphs at every corner of the solid-state landscape. The case has been examined in terms of solid-state energetics, rather than geometry or commercial X-ray software, by a trio of skeptical enquirers, ${ }^{[27]}$ with the predictable debunking of a number of claims (including one of $Z^{\prime}=11$ ). Mature researchers, more inclined to physics and thermodynamics than to Platonic super- or sub-symmetries, would be happy to read in a structural paper a sentence like "this material has been examined repeatedly by X-ray diffraction, picking several individuals from the crystallization batch, and a number of minor modifications and modulations have been found of what is, essentially, always the same crystal structure from the standpoint of stability, intermolecular interactions, and physical properties." Much more stimulating would be, at the other extreme, a sentence like "this material undergoes a reproducible modification at a certain temperature, and, in spite of the small structural difference, the high-temperature phase presents an evolution in .... (please enter a physical property here) so that the phase change may have an application as a molecular switch, a sensor, etc." This justifies a claim; in the former case, deposition of the modifications as new polymorphs only adds to confusion.

\subsubsection{Small Energies and Smaller Energy Differences}

There is a fundamental physical reason behind all this. Synthetic chemists make and break chemical bonds, which may be worth many hundreds, up to a thousand, $\mathrm{kJ} \mathrm{mol}^{-1}$. Total cohesive energies in the crystal of a regular organic molecule of medium size, say 30-50 atoms, may be no more than $80-150 \mathrm{~kJ} \mathrm{~mol}^{-1}$, summing up hundreds of intermolecular "bonds". One is speaking of different universes. What is even more problematic, differences between small energies are one order of magnitude smaller, so that crystal energy differences between polymorphs or even isomers may be no more than $5-20 \mathrm{~kJ} \mathrm{~mol}^{-1}$. This is why the exploration of phase space of organic crystals is often fruitless: not because of lack of accuracy of molecular simulation methods, but because of lack in accuracy by Nature in the preparation of its issues.

\subsubsection{The Curse of $50 \%$}

The availability of nearly a million crystal structure data for free analysis encourages the use of statistics to try to derive correlations in the structural chemistry of organic crystals. For example, in crystals with two molecules in the asymmetric unit, does the relationship between the two asymmetric partners have something special, say, a particularly high attachment energy? Yes, in about $50 \%$ of the cases. ${ }^{[28]}$ Are racemic crystals more dense or more stable than homochiral crystals? Yes, in about 50\% of the cases. ${ }^{[2]}$ In binary A...B cocrystals, is the relationship between the A...B molecular pair specially relevant in terms of cohesion energy? Yes, but only in about $50 \%$ of the cases. ${ }^{[30]}$ This state of affairs is the consequence of what has been stated in the two preceding paragraphs. The buildup of an organic crystal is a diffuse and multiform process, and it is always difficult to single out a special turning point, a special one-to-one correlation that may be held responsible for the outcome. A molecule with acceptor and donor terms will form a hydrogen bond in its crystal, with little or almost no exception, but as soon as there are more than one such terms, it soon becomes impossible to decide a priori what will be the result of competition. Once again, one is led to suspect that rather than coming from looking at crystals as they appear, the clue will come from the study of that crucial instant, when, in the growing crystal, embryos of one particular association motif will take over and start growing at the expense of all other possibilities. The key to crystal structure prediction and control is, in fact, control of the nucleation process. In this respect, the exponential growth of crystal structure databases is of little advantage; decades of experience have shown that conclusions reached by statistics on 10,000 crystal structures do not change over 1,000,000 crystal structures.

\section{Epilogue: From the Past to the Future}

This author and some other loafers who had nothing better to do were once discussing the following point: for good science, is it better to have a slow, but accurate, thinker, or a fast thinker, no matter if less accurate? JDD had been listening behind our shoulders, and characteristically, dropped in: "How about a fast and accurate thinker?" The present critical paper may seem somewhat biased towards the half-empty glass, but there is by now a numerous and well-endowed community of structural and solid-state chemists, slow or fast, that are making solid and steady progress. Structural chemistry is taking off with bright new ideas, followed by leveled plateaus of exhaustive computational and experimental developments, and is often landing on new chemical understanding and new applications, with the only proviso, as told in the opening of another JDD after-dinner story: "There are only three critical phases of commercial flight: takeoff, leveled flight, and landing."

Chemical science proceeds through long hours spent attending an instrument, watching a reaction vessel, or 
typing on a computer keyboard. An essential part of its development is, however, contact; human contact, in person, such as can only occur at meetings and conferences. For example, this author has been so far attending and contributing to two special occasions: in 1990, the Symposium in honor of J. D. Dunitz, Swiss Crystallographic Association (where JDD said, "I thank the organizers for inviting me to this meeting"); and in 2003, the Spring Meeting of the Swiss Chemical Society, in Honor of R. Ernst and J. D. Dunitz, both at the ETH in Zurich. Meetings like these, named after a person, are usually joyful occasions in which friends present their hommage to a friend. Other welcome events are small meetings with some twenty people bound by a common interest in a special topic, with a touch of family rehearsal (one such memorable occasion is pictured in Figure 5). On the contrary, large international congresses are often low-productivity events, in which long hours are spent wandering in busy corridors looking through a full morning of parallel sessions for the one lecture that could possibly be of interest. But they are also the only occasions to make friends that one will never be able to make over the electronic flash of a Facebook ghost, and to establish collaborations; or, in which a ten minute chat over a cup of mediocre coffee, or even a single sentence caught in a one hour talk, can provide the spark of new intuition. In this respect, one should really check his or her behavior, in order to maximize contact. I have heard American speakers giving talks at supersonic speed, in their broad southern twang, to an audience of students from central Africa at their first attendance of a foreign school. JDD's exemplary English, only biased by a faint Scottish accent, makes for easy understanding to all, except for his persistent tendency to wave his microphone instead of his pointer, with adverse consequence on both sound and sight.

Powerpoint presentation has been a Copernican revolution, but its dark side is the ease with which one can set up dazzling slides in bright colors and mesmerize the audience into expecting miracles. Very much apropos, starting his presentation of some smudgy acetate transparencies, JDD once apologized by showing a cartoon in which a gentleman, holding an open laptop, is kneeling in front of a lady whose punch line is: "Good powerpoint, Kevin, but the answer is no." Pope John XXII in 1317 fustigated the Alchemists by saying "spondent quod non exhibent" (they promise what they cannot maintain). So, please be sober. Ah, and don't forget to ask questions at the end of the talks; that's a vital part of the proceedings. In 1986, Ada Yonath was presenting early results on what would turn into a Nobel Prize some 30 years later. She said, "Our ribosome crystals were so unstable that we irradiated for just a few seconds, took quick plates, and then spent a lot of time indexing." At the end of the talk JDD stood up and said, "That's what I would call, shoot first, then ask questions."

We all walk on the shoulders of past giants. This author got to know Jack Dunitz as an inheritance of Jack's friendship with the author's mentor, Massimo Simonetta (Figure 6); Jack and Massimo, along with some other happy few, had been partners in science and beer drinking in Linus Pauling's group at Caltech in the 1950s. In the early decades of the past century, a number of pioneers (Figure 7) spent endless hours, days, and months (if not years) poring over long lists of numbers, calculating by hand structure factors in X-ray diffraction (Figure 8), and working out the structure of molecules in crystals without any help from computers. Nowadays, thanks to

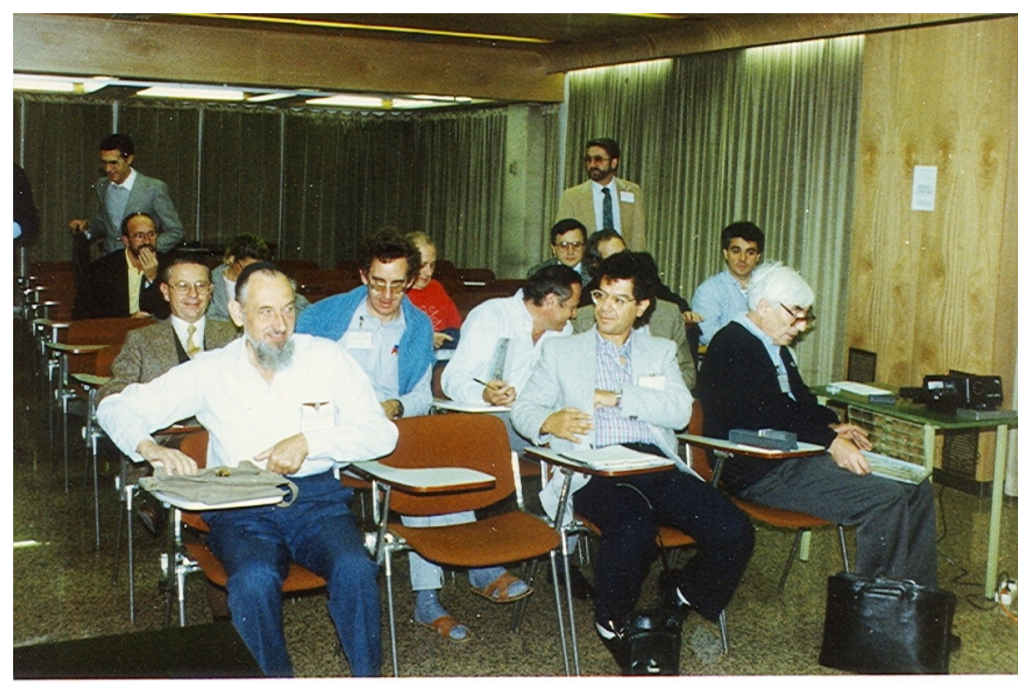

Figure 5. A bright future from a glorious past: at the Italian-Israeli meeting Steric and electronic effects on molecular crystalline structure, organized in Firenze, 1987, by the author and Joel Bernstein. Left to right: Gastone Gilli, Fred Hirshfeld, Hans-Beat Bürgi, Yithzak Apeloig, Sason Shaik, Jack Dunitz. Bürgi and Dunitz, from Bern and Zurich, were called in as "Swiss mercenaries". See www.iucr.org/gallery for more pictures. 

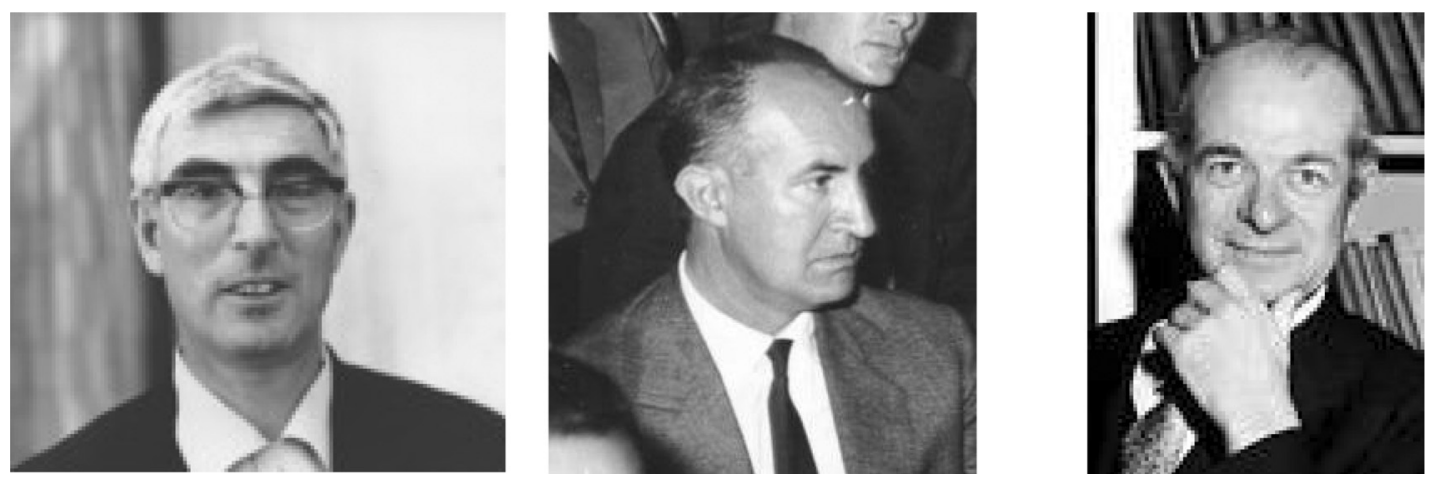

Figure 6. Left to right: an early Jack Dunitz; Massimo Simonetta (1920-1986); and Linus Pauling. There was an outstanding group of postdoctorals at CalTech in the 1950s, which included, among others, Martin Karplus.

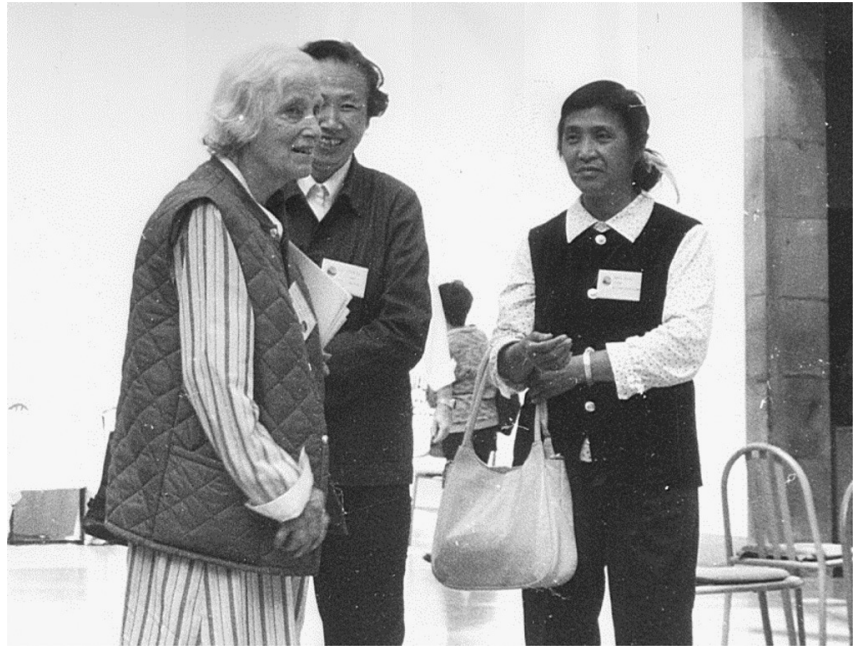

Figure 7. Pioneers: Dorothy Crowfoot Hodgkin, with whom Jack Dunitz spent a momentous postdoctoral, seen with her Chinese chaperones at a Conference in Beijing, 1986. See www.iucr.org/gallery, 1986, for more pictures from the author's personal archives.

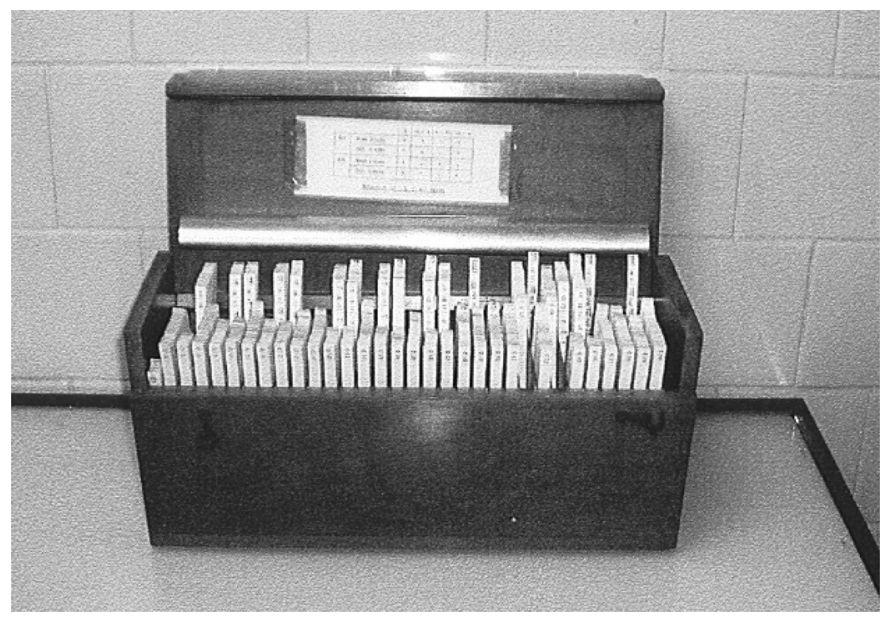

Figure 8. A set of Beevers-Lipson strips for calculating X-ray structure factors by hand, still available at the Dipartimento di Chimica in Milano. Readers who may need to use the set, because of computer breakdown, are welcome to contact the author. those who developed the methods, the determination of the structure of an organic crystal is almost a matter of minutes, and never before in its history has chemistry changed so fast in so little time as when the clear, incontrovertible results of X-ray crystal and molecular structure determinations became readily available. The opportunity of seeing the size and shape of molecules, marked by countless Nobel prizes to crystallographers, along with the development of reliable quantum chemical methods to determine the shape of their electron density, marked by John Pople's Nobel prize, has opened an entirely new era: the era in which properties of macroscopic bodies can be predicted at least to a good extent from the structure of the constituting molecules. People holding today in the palm of their hands one of those shiny gadgets that are, in fact, at the same time, a powerful computer and a highly performing television screen, owe something to the efforts of those pioneers.

\section{References}

[1] P. Seiler, W. B. Schweizer, J. D. Dunitz, Acta Crystallogr. 1984, $B 40,319$.

[2] From Groucho Marx on: "Mr. Marx, there's a gentleman for you, with a moustache", "Tell him I already have one".

[3] Most of these stories have been told in a regular afterdinner feature at the NATO ARW Crystals: Supramolecular Materials, in Sestri Levante, 1995, organized by the author and Giuseppe Filippini.

[4] Gloss: for ladies, a sort of lipstick; for philosophers, a note made in the margin of a text. Famous example: Fermat's last theorem.

[5] A scholium is a short treatise in the margin; see Cicero, Epist. ad Att., XVI, 7, 3.

[6] See, for some discussion, A. Gavezzotti, Molecular Aggregation, $2^{\text {nd }}$ Edition, Oxford University Press, Oxford, 2013, Chapter 13.

[7] D. W. Oxtoby, Acc. Chem. Res. 1998, 31, 91.

[8] Parts of the development of rhetoric (Medieval eloquence): argumentatio, a statement; confirmatio and confutatio, its two consequents (scholars were often taught to sustain both, an excellent intellectual exercise). 
[9] "Trembling deeply, so he gave voice to fate": Proteus the diviner, in Vergil's Georgic IV, $452 \mathrm{ff}$, poetry unsurpassed across the millennia.

[10] A. S. Myerson, B. L. Trout, Science 2013, 341, 855.

[11] For part of the Koerner story, see: F. Demartin, G. Filippini, A. Gavezzotti, S. Rizzato, Acta Crystallogr. 2004, B60, 609.

[12] A. Gavezzotti, Cryst. Growth Des. 2013, 13, 3801.

[13] M. Salvalaglio, T. Vetter, F. Giberti, M. Mazzotti, M. Parrinello, J. Am. Chem. Soc. 2012, 134, 17221.

[14] Another exercise of medieval rhetoric; it consists of writing a prescribed number of pages on a given theme. Curiously, this kind of exercise has survived to our school days.

[15] J. D. Dunitz, ChemBioChem 2004, 5, 614.

[16] Examples chosen at random: a) D. Chopra, T. N. Guru Row, CrystEngComm 2011, 13, 2175; b) H. Omorodion, B. Twamley, J. A. Platts, R. J. Baker, Cryst. Growth Des. 2015, $15,2835$.

[17] A. Gavezzotti, CrystEngComm 2013, 15, 4027.

[18] A. Gavezzotti, Acc. Chem. Res. 1994, 27, 309.

[19] J. D. Dunitz, Chem. Commun. 2003, 545.

[20] A. Gavezzotti, J. Am. Chem. Soc. 1991, 113, 4622.

[21] D. A. Bardwell, C. S. Adjiman, Y. A. Arnautova, E. Bartashevich, S.X. M. Boerrigter, D. E. Braun, A. J. CruzCabeza, G. M. Day, R. G. Della Valle, G. R. Desiraju, B. P. van Eijck, J. C. Facelli, M. B. Ferraro, D. Grillo, M. Habgood, D. W. M. Hofmann, F. Hofmann, K. V. J. Jose, P. G. Karamertzanis, A. V. Kazantsev, J. Kendrick, L. N. Kulesho- va, F. J. J. Leusen, A. V. Maleev, A. J. Misquitta, S. Mohamed, R. J. Needs, M. A. Neumann, D. Nikylov, A. M. Orendt, R. Pal, C. C. Pantelides, C. J. Pickard, L. S. Price, S. L. Price, H. A. Scheraga, J. van de Streek, T. S. Thakur, S. Tiwari, E. Venuti, I. K. Zhitkov, Acta Crystallogr. 2011, B67, 535 (a 41-author paper).

[22] I. C. Paul, D. Y. Curtin, Chem. Rev. 1981, 81, 525.

[23] For a recent mise au point, although with largely unwarranted views about the merits of the cited people, some of which (like this author) have never had anything to do with crystal engineering, see E. R. T. Tiekink, Chem. Commun. 2014, 50, 11079.

[24] J. Bernstein, Cryst. Growth Des. 2011, 11, 632.

[25] S. Chen, I. A. Guzei, L. Yu, J. Am. Chem. Soc. 2005, 127, 9881.

[26] A. L. Bingham, D. S. Hughes, M. B. Hursthouse, R. W. Lancaster, S. Tavener, T. L. Threlfall, Chem. Commun. 2001, 603.

[27] J. Bernstein, J. D. Dunitz, A. Gavezzotti, Cryst. Growth Des. 2008, 8, 2011.

[28] A. Gavezzotti, CrystEngComm 2008, 10, 389.

[29] A. Gavezzotti, S. Rizzato, J. Org. Chem. 2014, 78, 4909.

[30] V. Colombo, L. LoPresti, personal communication from work in progress.

Received: April 4, 2016

Published online: June 24, 2016 